\title{
Regulation of Organ Growth by Morphogen Gradients
}

\author{
Gerald Schwank and Konrad Basler \\ Institute of Molecular Biology, University of Zurich \\ Correspondence: konrad.basler@molbio.uzh.ch
}

\begin{abstract}
Morphogen gradients play a fundamental role in organ patterning and organ growth. Unlike their role in patterning, their function in regulating the growth and the size of organs is poorly understood. How and why do morphogen gradients exert their mitogenic effects to generate uniform proliferation in developing organs, and by what means can morphogens impinge on the final size of organs? The decapentaplegic (Dpp) gradient in the Drosophila wing imaginal disc has emerged as a suitable and established system to study organ growth. Here, we review models and recent findings that attempt to address how the Dpp morphogen contributes to uniform proliferation of cells, and how it may regulate the final size of wing discs.
\end{abstract}

\section{THE RELATIONSHIP BETWEEN PATTERNING AND GROWTH}

$\mathrm{H}^{2}$ ow form and size are regulated during animal development is one of the most fundamental questions in biology. Two essential underlying mechanisms, patterning and growth, are tightly linked during embryogenesis.

First evidence for such a link came from regeneration studies on cockroach legs. When two fragments with different positional values are juxtaposed, intercalary growth occurs and the gap is filled with tissue of the transitional positional values (Bohn 1976; French et al. 1976). This mechanism of intercalary growth, which occurs in various systems, suggests that pattern formation regulates cell proliferation. Is there really a strict hierarchical relationship between these processes in animal development, with patterning acting upstream of growth?
Prima facie it seems that this is indeed the case, as there are many instances in which patterning processes appear to define units that intrinsically carry information about their final size. Some of the strongest evidence for this partitioning into units of growth control comes from studies of the developing Drosophila wing. Here, patterning first divides the developing organ into an anterior (A) and a posterior $(\mathrm{P})$ compartment. The two compartments each obtain an intrinsic growth program and know when to stop growing independently from each other (Simpson 1976; Day and Lawrence 2000; Martin and Morata 2006). Further evidence stems from studies of the developing chick wing, in which a patterning process defines cartilaginous elements, which then follow their intrinsic growth programs (Wolpert 1981). On closer inspection, however, it becomes apparent that these

Editors: James Briscoe, Peter Lawrence, and Jean-Paul Vincent

Additional Perspectives on Generation and Interpretation of Morphogen Gradients available at www.cshperspectives.org

Copyright (C) 2010 Cold Spring Harbor Laboratory Press; all rights reserved; doi: 10.1101/cshperspect.a001669

Cite this article as Cold Spring Harb Perspect Biol 2010;2:a001669 
examples merely reflect the iterative nature of development, a process during which the organism is progressively subdivided into smaller units in a temporally hierarchical order. The establishment of the main body axes, for example, predetermines the anlagen of many organs, which in turn develop as individual units that are further subdivided into subunits, and so on. Quite naturally, this leads to apparently hierarchical relationships, in which all events within a unit (including growth) occur downstream of the developmental processes (including patterning) that took place during the previous steps in development.

The nature of the relationship between growth and patterning within one "hierarchical step" is therefore the most intriguing. As mentioned above, intercalary growth experiments provide evidence that patterning regulates cell proliferation. The opposite also seems to be the case, i.e., that proliferation of cells and the resulting growth of a tissue is a prerequisite for patterning, as recently shown in experiments addressing digit patterning in the chick wing (Towers et al. 2008). This concept of a mutual requirement is supported by the finding that the same molecules that regulate patterning, namely morphogens, simultaneously regulate proliferation and growth. Traditionally, the term morphogen (from the Greek morphê shape and genesis creation) has been used in the context of a specific patterning concept, i.e., that gradients of morphogen substances execute patterning in a concentrationdependent manner. However, the first molecular identification of morphogens in Xenopus already showed that these substances are also involved in growth control: They turned out to belong to previously known families of growth factors, the fibroblast growth factor (FGF) and the transforming growth factor-beta (TGF-beta) families (Kimelman and Kirschner 1987). This general concept, that morphogens are patterning agents and growth factors, is supported by several other studies in different organisms. Prime examples are Sonic hedgehog (Shh) in mouse and chick limb development (reviewed in Bénazet and Zeller 2009), and Decapentaplegic (Dpp) in Drosophila wing development (reviewed by Affolter and Basler 2007).

\section{UNIFORM PROLIFERATION, PRECISION, AND DEVELOPMENTAL ROBUSTNESS}

Gradients of morphogens specify cell fates within a developing organ by activating target gene expression in a concentrationdependent-and hence position-specificmanner. If these morphogens also exert their role as mitogens in a concentration-dependent manner, one would expect proliferation rates to differ within a morphogen's realm of action as a function of the distance to the source, i.e., higher proliferation levels close to the site of morphogen production, and lower proliferation levels further away. Studies on organ growth and proliferation suggest that this is not the case. During vertebrate limb bud formation, for example, the morphogens Shh and fibroblast growth factors (FGFs) control the establishment of the AP and PD axes, as well as cell proliferation and expansion of the limb bud (Ten Berge et al. 2008; Towers et al. 2008; Zhu et al. 2008). Although these morphogens form concentration gradients, they drive proliferation uniformly within early stages of limb formation. A further example is provided by the Drosophila wing imaginal disc. Cells proliferate uniformly during larval development, the time during which their AP and DV fates are patterned by the gradient morphogens Decapentaplegic (Dpp) and Wingless (Wg), respectively (Milan et al. 1996). Thus, also in this system, morphogens drive uniform proliferation despite being distributed in gradients. Before we discuss different models that try to resolve the conundrum of how a growth factor gradient can lead to uniform proliferation, we first examine possible advantages of uniform proliferation during organ development.

During development, the organ is exposed to a certain "developmental noise": Fluctuations of environmental factors, genetic variability, and stochastic differences can lead to variations in gene expression levels between individuals. Despite such fluctuations, the outcome of organ development is very precise 
and reproducible, indicating that the molecular mechanisms regulating patterning and growth of organs must be robust and able to buffer these variations. Does uniform cell proliferation during organ patterning contribute to reach a high degree of precision and robustness? As we show in Figure 1, when coupled to proper scaling of the morphogen gradient readout, uniform proliferation appears to allow an increased flexibility regarding the time point of halting patterning and freezing the expression boundaries of target genes (Fig. 1A,C, Box 1). As discussed below, this could on the one hand contribute to precision in specifying the expression domains of target genes (i.e., patterning), and on the other hand contribute to robustness by buffering fluctuations in the levels of morphogen production.

How can flexibility in the time point of the patterning decision contribute to the precision and robustness of Drosophila wing patterning? Recent experimental observations and theoretical considerations indicate that the Dpp morphogen gradient provides maximal precision in positional information at a definedbut-limited distance away from the source (Bollenbach et al. 2008). Moreover, assuming an exponentially decreasing concentration gradient, fluctuations in morphogen production rates would lead to a shift in cell fate boundaries if these boundaries are distant from the source of Dpp production (Eldar et al. 2003). It may thus be advantageous to start defining the nested expression domains of different Dpp target genes, such as those of optomoter-blind $(o m b)$ and spalt (sal), at early, staggered time points during development, when the respective expression boundaries are still close to the Dpp source. The subsequent expansion of a predefined arrangement of nested target-gene expression domains would then critically rely on a uniform pattern of cell proliferation for correct proportions to be conserved.

It is important, however, to point out that the advantages of uniform proliferation for increased precision and robustness mentioned here are purely speculative, and not based on experimental observations. In fact, so far there is no evidence in the Drosophila wing system that the sal or omb expression domains become fixed at any time point during development. Several experiments actually indicate that the opposite might be the case, and that cells can change their fate if Dpp signaling is altered late in development (Nellen et al. 1994; Singer et al. 1997). Thus, further studies on cell fate specification and scaling of the morphogen gradients during wing disc development will be essential to experimentally address the above considerations. Indeed, such experiments might also provide alternative explanations for an advantage of uniform proliferation during organ development. One such alternative explanation could be the existence of an optimal proliferation rate (highest speed of proliferation at which normal cellular functioning is not affected, and physiological problems do not occur). To reach the final size of an organ as fast as possible, without losing robustness in correct development, all cells would have to divide at the same, optimal proliferation rate.

\section{HOW CAN A GRADIENT OF A GROWTH FACTOR ACCOUNT FOR UNIFORM PROLIFERATION?}

In the following two sections, we address the question of how a growth factor that is distributed in a gradient can account for uniform proliferation. We first discuss four general groups of models that could explain uniform proliferation, and then in the next section we examine models which specifically try to explain how the Dpp morphogen gradient in the wing imaginal disc can lead to uniform proliferation (Fig. 3).

The first group of models are the "memory models." In such models a morphogen has a sequential mode of action. First, the morphogen gradient desensitizes target cells in a way that cells located close to the morphogen source are less sensitive to the mitogenic action of the morphogen, whereas cells further away become more sensitive. Cells memorize the levels of the initial signal, and behave accordingly during the proliferation period later in development. Organ growth would then be uniform despite graded morphogen 
G. Schwank and K. Basler

A No scaling - uniform proliferation

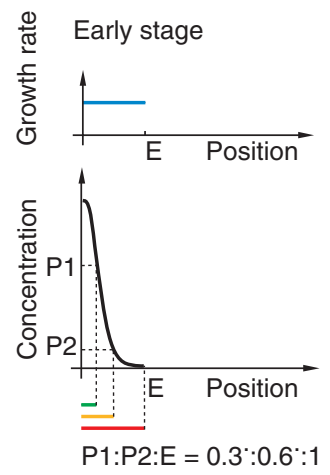

Determination
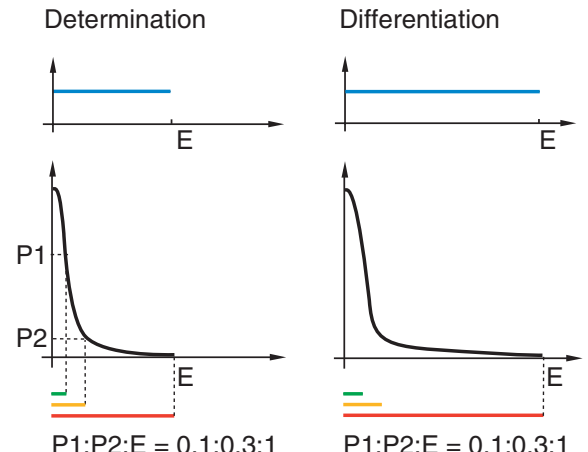

B Scaling - no uniform proliferation
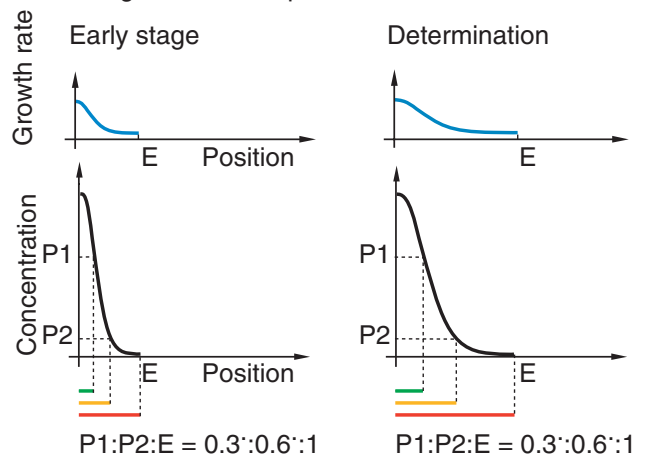

Differentiation

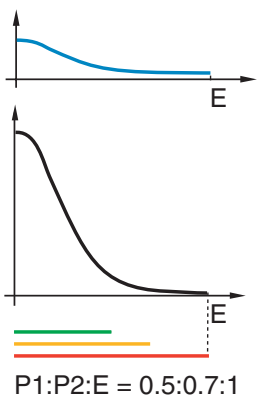

C Scaling - uniform proliferation

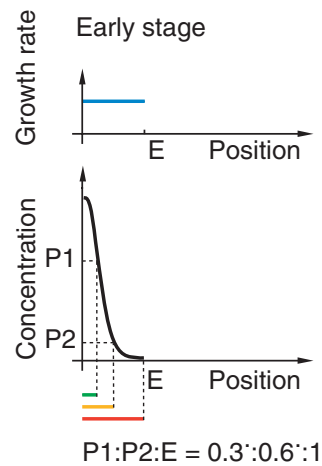

Determination

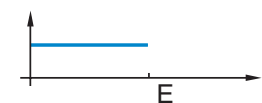

Differentiation
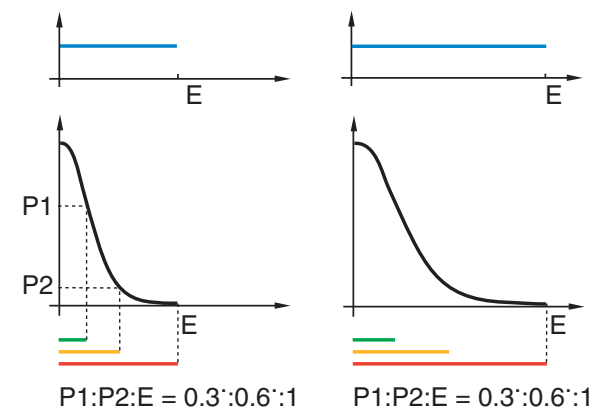

Figure 1. Uniform growth together with scaled morphogen gradients allows determination of cell fates at different time points without losing the right proportions. $(A)$ The case in which growth is uniform, but the morphogen gradient is not scaled. This leads to a change in the proportions of target gene expression domains. Thus, in this situation, the final proportions strictly depend on the precise time-point of cell fate determination. (B) A morphogen gradient scaled to organ size, but driving nonuniform growth. The expression domains of target genes are in proportion only until cell fate determination. Thus, also here, the time point of cell fate determination cannot be shifted without a change in the final proportions. (C) A morphogen gradient scaled to organ size together with uniform growth. In this situation, the expression domains of target genes keep the right proportions independent of the time-point of cell fate determination. Note that the values of the proportions are not based on experimental data. (P1 and P2) Threshold levels for target gene 1 and 2, (E) endpoint of the organ. 


\section{BOX 1: EVIDENCE FOR UNIFORM PROLIFERATION AND A SCALING IN THE DROSOPHILA WING DISC}

Support for uniform proliferation:

Evidence for uniform proliferation in the Drosophila wing imaginal disc comes from studies using mitotic markers (BrdU staining for cells in S-phase and pH-H3 staining for cells in $\mathrm{M}$-phase) as well as from the analyses of genetically neutral cell clones (Milan et al. 1996; Martin-Castellanos and Edgar 2002; Schwank et al. 2008).

Support for scaling:

Studies on the "critical weight" in Drosophila, early in the last century, support the existence of "scaling," i.e., adaptation of the pattern to changes in size. The critical weight is defined as the weight at which larvae can continue to develop into adults even if food is completely withdrawn. Drosophila larvae, after reaching the critical weight, continue to grow if they are cultured on rich food. If starved, however, these larvae stop growing and develop into adults of severely decreased size. Despite these differences in size, the pattern and proportions of these animals are normal (Beadle et al. 1938). Moreover, a recent study additionally suggests a scaling mechanism by showing that the relative distances between the wing veins remains constant and in proper proportions to the overall wing size (Bollenbach et al. 2008). This maintenance of proportions can already be observed in gene expression patterns, such as the expression domain of the Dpp target gene spalt (sal), which sets the position of vein two (Sturtevant et al. 1997; Bollenbach et al. 2008). It is important to point out, however, that the mechanism underlying scaling in this system is not known yet. In principle, scaling could be accomplished either by continuously extending the morphogen gradient and thereby adjusting its shape relative to the growing tissue, by continuously adapting the response of cells to compensate for the increase in tissue size, or by defining target gene expression domains early in development followed by a phase of uniform expansion of the tissue during which cells maintain the expression states of these genes.

levels, because proximal cells exposed to higher concentrations of the morphogen are less sensitive, and distal cells exposed to lower concentrations of the morphogen are more sensitive. As a consequence, the actual proliferative response of the cells to the gradient becomes uniform. Importantly, cells show different sensitivities only for the mitogenic action of the morphogen, but not for the patterning response.

A second class can be summarized as "threshold models." According to these models, morphogen-dependent proliferation occurs wherever morphogen concentrations are above a certain value. Thus, in these models, there is an all-or-none response to the morphogen gradient with respect to proliferation. Uniform proliferation within an entire organ would occur if the levels of the morphogen are above the threshold throughout the organ. The models are therefore similar to the "French flag" model of patterning, in which the morphogen gradient defines gene expression boundaries via thresholds, but within the boundaries the gene expression levels are uniform (Wolpert 1969).

A third group are the "gradient models." In such models, proliferation depends on the slope of the morphogen gradient (Gelbart 1989; Lawrence and Struhl 1996). Here, it is not the absolute morphogen level an individual cell is exposed to that determines its proliferation behavior, but rather the relative differences vis-à-vis neighboring cells. Such models demand a molecular mechanism that allows cells to compare the morphogen levels they are exposed to with those of adjacent cells.

The fourth group comprises the "multiple growth parameter models." These models assume that growth is not only regulated by a morphogen, but in combination with "complementary" growth modulators. Such growth modulators regulate growth differently in distinct regions of the organ, and it is the 
combination of their input with that of the morphogen that ensures uniform proliferation. In its simplest forms, a growth repressor would be distributed in a gradient parallel to that of the morphogen, or a growth activator in a counter-gradient. The establishment and activities of these additional growth modulators could be dependent on, or arise independently of, the morphogen. Moreover, such "complementary" growth modulators can be of different nature: They could be morphogens themselves, growth factors without patterning function, or even nonmolecular factors, such as mechanical influences.

\section{THE DPP GRADIENT DRIVES UNIFORM PROLIFERATION IN THE DROSOPHILA WING DISC}

An experimentally amenable system to explore a situation in which a gradient can generate uniform proliferation is the Dpp morphogen gradient in the wing imaginal disc. This disc is the precursor organ for the adult wing and arises as an epithelial, saclike structure at the end of embryogenesis. During subsequent larval stages (first until third instar stage), the wing disc is patterned and gains mass. It grows from about 50 to 50,000 cells with an average cell cycle time of around $8 \mathrm{~h}$ (Bryant and Levinson 1985). Although cell divisions occur in clusters of cells, proliferation within the overall disc is uniform (Milan et al. 1996). The morphogenesis of the disc into the adult wing takes place during metamorphosis in the pupal stage, which follows the larval stages. Key players for patterning the disc are the morphogens Wingless (Wg, along the DV axis) and Dpp (along the AP axis). The AP patterning and subsequent specification and formation of the wing veins has been described in detailreviewed in (Affolter and Basler 2007; Blair 2007) and illustrated in Figure 2. Dpp is expressed in the developing wing disc in a narrow stripe of cells in the A compartment along the AP compartment boundary. Because it is a secreted ligand, Dpp moves from its source of production into the more lateral areas, forming a concentration gradient and

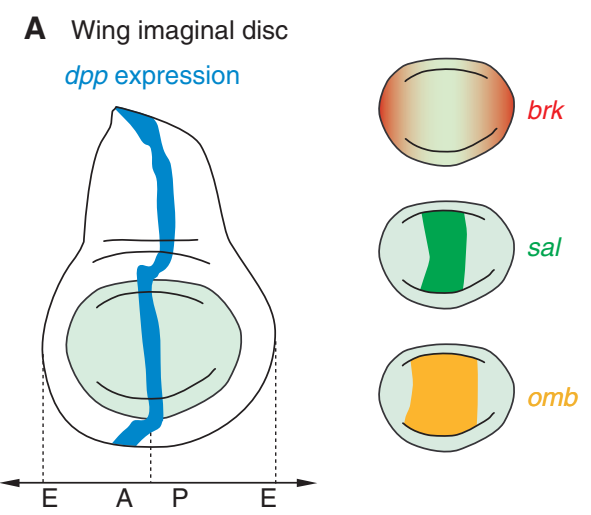

B Dpp gradient

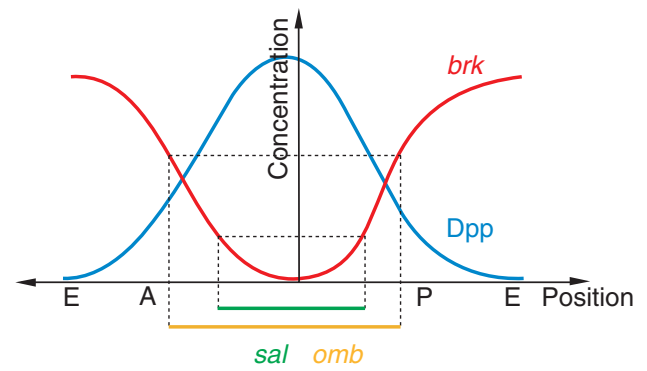

Figure 2. The Dpp gradient in the developing wing imaginal disc of Drosophila serves as a model to study organ patterning and growth. $(A)$ Expression domains of $d p p$, brk, sal, and omb. (B) Dpp is secreted from its site of production in the center of the disc and spreads into the A and P compartment, establishing a gradient with highest levels in the center and lowest in the periphery. brk transcription is gradually repressed by Dpp signaling activity, leading to a $b r k$ gradient inverse to the Dpp gradient. Brk levels are important to set the expression boundaries of sal and $o m b$. omb is repressed by high levels of Brk, and sal by lower levels. Consequently, omb is expressed in a broader domain than sal. (A) Anterior compartment, $(\mathrm{P})$ posterior compartment.

exerting a long-range organizing effect in both compartments (Entchev et al. 2000; Teleman and Cohen 2000). Dpp negatively regulates the expression of the transcriptional repressor Brinker (Brk), creating a gradient of brk expression that is reciprocal to the Dpp gradient. Brk regulates in a concentration dependent manner the nested expression domains of the genes sal and $o m b$, which position the longitudinal veins along the AP axis of the wing (Sturtevant et al. 1997; Campbell and 
Tomlinson 1999; Jazwinska et al. 1999; Minami et al. 1999; Marty et al. 2000; Sivasankaran et al. 2000; Cook et al. 2004).

Several studies indicate that Dpp not only regulates patterning, but also plays a role in growth regulation of the Drosophila wing disc. Loss- and gain-of-function studies in the wing disc show that it is a positive growth regulator and required for the uniform cell proliferation occurring in the disc (Spencer et al. 1982; Capdevila and Guerrero 1994; Zecca et al. 1995; Burke and Basler 1996; Adachi-Yamada et al. 1999; Martin-Castellanos and Edgar 2002; Rogulja and Irvine 2005). Moreover, several studies show that Dpp regulates growth, just like patterning, via Brk (Campbell and Tomlinson 1999; Martin et al. 2004; Schwank et al. 2008). The wing imaginal disc is a model system that is uniquely suited to study how a morphogen gradient can account for uniform proliferation. Notably, sophisticated genetic tools in Drosophila permit us to experimentally modify the Dpp gradient and thus test the different models proposed to account for uniform proliferation. In the following section, we first discuss models that were already confuted by experimental findings, and then describe an attempt to incorporate these new findings.

\section{The Memory Model}

According to the memory model, the Dpp gradient at the onset of wing disc development would program the cells of the disc along the AP axis to acquire different sensitivities towards a mitogenic activity of Dpp-such that lateral cells become more sensitive relative to medial cells. Thus, during the growth period of the disc, the Dpp morphogen gradient would lead to uniform proliferation despite elevated Dpp activity in medial compared with lateral regions (Fig. 3A). One experimental situation appears to support this model. When Dpp is expressed ubiquitously, from midlarval stages onwards, wing discs overgrow because of excessive proliferation of laterally situated cells (Nellen et al. 1996; Martin-Castellanos and Edgar 2002; Rogulja and Irvine 2005); therefore, lateral cells are apparently more sensitive than medial cells vis-à-vis Dpp's growth-promoting effects. However, in light of very recent observations, the memory model can no longer be upheld. If all cells of the wing disc are subjected to equal, high levels of Dpp pathway activity, from the very beginning of disc development onwards, lateral cells will still proliferate faster than medial cells. Evidently, different sensitivities to the mitogenic action of Dpp exist (Schwank et al. 2008) despite exposure to equal levels of Dpp early on, but this difference is not related to an early exposure to a gradient of Dpp.

\section{The Threshold Model}

According to the threshold model, Dpp would drive uniform proliferation because its activity exceeds a certain minimal level throughout the disc (Fig. 3B). There is, however, experimental counter-evidence against such a scenario in the wing disc. As mentioned above, ectopic uniform activation of Dpp leads to overgrowth of the discs (Capdevila and Guerrero 1994; Burke and Basler 1996); moreover, lateral clones with constitutively active Dpp signaling grow faster than wild-type clones (MartinCastellanos and Edgar 2002). The threshold model would predict, however, that once the marginal value is surpassed, differences in Dpp levels should no longer matter.

\section{The Gradient Models}

The gradient model for growth regulation, applied to the wing disc, predicts that cells compare the levels of Dpp they are exposed to with those of their neighbors. When the differences between these levels are sufficiently high, i.e., in which the gradient of Dpp is sufficiently steep, cells proliferate (Fig. 3C) (Day and Lawrence 2000). However, this model would predict proliferation to stop where these differences are zero, such as in discs with ubiquitous Dpp expression, and this is not the case. This discrepancy led to a refinement of the model (Rogulja and Irvine 2005): During early wing disc development, lateral cells may become differently programmed, such that they not only 
G. Schwank and K. Basler

A Memory model

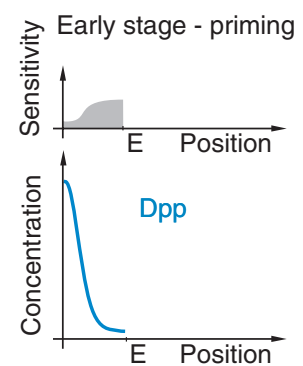

C Gradient model

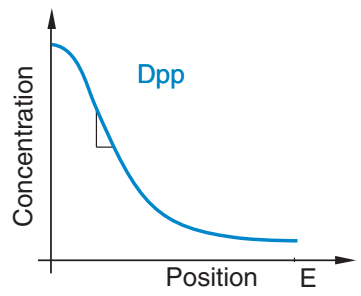

F Steamroller model

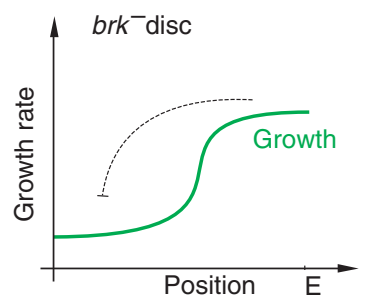

B Threshold model

Later stage - proliferation

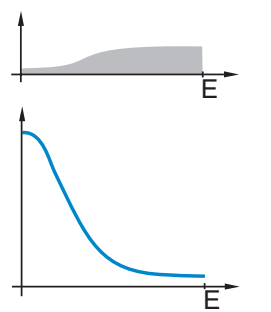

D Modified gradient model

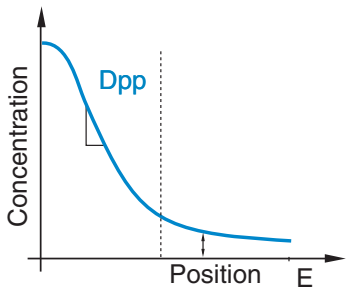

E Inhibitor model
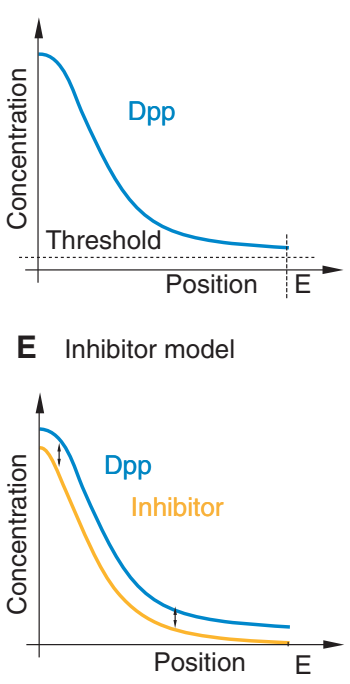

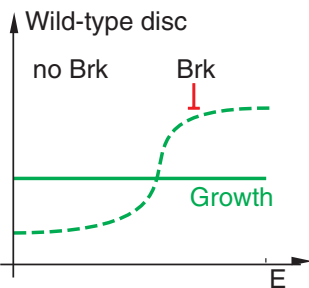

Figure 3. Models addressing how the Dpp gradient can account for uniform growth within the wing disc. (A) Cells are primed for different sensitivity to the mitogenic activity of Dpp, which leads to uniform proliferation during the growth period. $(B)$ Dpp drives proliferation at constant levels above the threshold value, which is reached within the entire wing disc. $(C)$ In the gradient model, the slope of the Dpp gradient drives proliferation. $(D)$ A modified version of the gradient model, in which the disc is subdivided into two regions, the medial and the lateral regions. In the medial region, where the Dpp gradient is sufficiently steep, cells proliferate in response to the gradient, and in the lateral region, where the gradient becomes very shallow, cells proliferate in response to absolute Dpp levels. (E) A growth inhibitor in parallel to Dpp evens out high differences of Dpp signaling along the AP axis. $(F)$ In this model, lateral cells have a growth advantage compared with medial cells, and the Dpp/Brk system is needed to curb lateral proliferation. If absent, lateral overproliferation by an unknown mechanism inhibits growth in the medial area.

react to the steepness of the Dpp gradient, like medial cells, but also to the absolute local Dpp levels-even when these are uniform (Fig. 3D). This model is supported by the following experimental observations: Activation of the Dpp pathway in clones, by expression of $\mathrm{Tkv}^{\mathrm{QD}}$ (a constitutively active Dpp receptor), leads to a transient increase of proliferation at the clone margin, which was attributed to the large difference in Dpp pathway activity between adjacent cells on either side of the clone margins. Further support was derived from the experiment with uniform activation of the Dpp pathway, which leads to overproliferation of lateral cells and reduced proliferation in medial cells (Rogulja and Irvine 2005). 
Regulation of Organ Growth by Morphogen Gradients

However, a recent study found that juxtaposition of cells with distinct Dpp signaling levels is not necessary to drive proliferation, because uniform expression of $\mathrm{Tkv}^{\mathrm{QD}}$ exclusively in the medial area leads to normal growth within the entire disc (Schwank et al. 2008). Additional experiments indicate that the reduced proliferation observed in the medial region of discs with uniformly high Dpp signaling is not because of the loss of graded Dpp signaling levels, but because of a growth-retarding signal from the overproliferating lateral cells (Schwank et al. 2008). Taken together, these new results strongly challenge the validity of a gradient model.

\section{The Inhibitor Model}

One model that is based on a growth input in addition to that of Dpp is the inhibitor model. This model postulates that a growth inhibitor forms gradient parallel to Dpp, resulting in a net growth stimulus that is similar for all cells along the AP axis (Fig. 3E) (Serrano and O'Farrell 1997). The expression of the growth inhibitor could be controlled by Dpp or arise independently of Dpp. If it would occur downstream of Dpp, homogeneous activation of the Dpp signaling pathway should lead to homogenous growth. As described above, however, this is not the case (Rogulja and Irvine 2005; Schwank et al. 2008). Should the inhibitor expression arise independent of Dpp, a likely scenario would be that it is controlled by $\mathrm{Hh}$ signaling, which occurs in a narrow territory along the AP axis and directly or indirectly regulates the expression of all known AP patterning genes, including $d p p$ itself. However, uniform activation of the $\mathrm{Hh}$ signaling pathway in the anterior half of the wing disc leads to overgrowth and higher proliferation rates in the anterior lateral region (Basler and Struhl 1994). The absence of any sign on an inhibitor that curbs the ectopic activity of Dpp in this situation makes it unlikely that the hypothetical inhibitor is controlled by hedgehog (Hh). Moreover, no such inhibitor has ever been identified, making the inhibitor model speculative.
The Steamroller Model

Another model based on multiple growth inputs is the "steamroller model" (Fig. 3F) (Schwank et al. 2008). In discs in which the Dpp/Brk system has been genetically removed, the authors noted that a nonuniform proliferation pattern resulted-with increased growth laterally and decreased growth medially. They interpreted this as evidence for an additional growth input independent to that of Dpp, and that the main role of the Dpp/ Brk system is to level inherent growth inequalities like a steamroller (Fig. 3F). What could be the molecular nature of the growth activator in the lateral areas of the wing disc? One possibility would be a diffusible growth activator emanating from the interface between cells of the peripodial membrane and the disc proper. Another type of explanation invokes mechanical forces that act on growth. Two such models (see "The Dpp Gradient and the Final Size Question") postulate that high amounts of growth factors in the central region create certain mechanical forces within the disc, which then feedback on growth and lead to uniform proliferation (Shraiman 2005; Aegerter-Wilmsen et al. 2007; Hufnagel et al. 2007). In discs with experimentally expanded Dpp signaling activity, these mechanical forces would differ, resulting in an abnormal influence on growth and a nonuniform proliferation pattern. As both mechanical forces models not only try to explain uniform proliferation but also final size of the wing disc, they will be discussed later in the section "The Dpp Gradient and the Final Size Question.”

\section{DPP'S CONNECTION TO THE CELL CYCLE}

Organ growth can be principally regulated by controlling (1) the size, (2) the survival, or (3) the proliferation of cells. By which of these means does Dpp regulate wing disc growth? Changes in cell size are not observed when Dpp levels are altered, indicating that Dpp does not regulate organ growth by affecting cell size (Martin-Castellanos and Edgar 2002). Moreover, experimentally induced polyploidy, 
which results in increased cell size, does not alter the final size of the wing imaginal discs (Santamaria 1983; Weigmann et al. 1997). A role in cell survival has been indicated by $d p p$ loss-of-function and $b r k$ gain-of-function clones. Such cells are eliminated from wing imaginal discs (Burke and Basler 1996; Adachi-Yamada et al. 1999; Moreno et al. 2002). Supporting this idea, $b r k$ overexpression leads to JNK-mediated apoptosis, and the elimination of brk gain-of-function clones can be prevented by blocking apoptosis (Moreno et al. 2002). Cell-biological analysis of clones with reduced Dpp signaling indicates that these cells might be extruded because of the loss in tissue integrity caused by the vast differences of Dpp signaling levels between cells inside and outside surrounding the clones (Gibson and Perrimon 2005; Shen and Dahmann 2005). Thus, at least partially, Dpp acts as a survival factor by promoting epithelial integrity. Besides the evidence for Dpp promoting cell survival, proliferation assays (staining for cells in S- or M-phase and in clonal analyses) indicate a strong relationship between Dpp signaling levels and proliferation rates. Ectopic Dpp signaling in the lateral regions of the wing disc, where endogenous levels are very low, lead to an increase in proliferation levels (Macdonald and Goldstein 1999; MartinCastellanos and Edgar 2002; Martin et al. 2004; Rogulja and Irvine 2005; Schwank et al. 2008).

Most of the molecular components linking Dpp signaling activity to the cell cycle and the apoptosis machinery, however, remain to be identified. Several studies show that Dpp uses the transcriptional repressor Brk for regulating growth (Jazwinska et al. 1999; Martin et al. 2004; Schwank et al. 2008). The downstream machinery connecting Brk to the cell cycle, however, remains obscure. Only a few transcriptional target genes of Brk are known, and only for one, vestigial $(v g)$, has an influence on cell proliferation and survival been described (Kim et al. 1996; Campbell and Tomlinson 1999; Van de Bor et al. 1999).

$\mathrm{Vg}$ functions as a transcription factor and is expressed within the wing pouch of the wing imaginal disc (Kim et al. 1996). Cells in the wing pouch lacking vg function undergo cell death, which causes a loss of the corresponding wing structures in the adult (Agrawal et al. 1995). The phenotype of vg clones resembles that of clones which lack Dpp input or overexpress brk (Burke and Basler 1996; Kim et al. 1996; Adachi-Yamada et al. 1999; AdachiYamada and O'Connor 2002; Moreno et al. 2002; Baena-Lopez and Garcia-Bellido 2006), and overexpression of $v g$ can prevent the elimination of brk-expressing clones (A. Smith and K.B., unpublished data). However, vg expression is not only regulated by Dpp, but also by the activities of the Wg and Notch signaling pathways (Couso et al. 1995). Recently, it has been shown that the $v g$ expression domain forms because of an autoregulatory feed forward mechanism, which relies on short range Notch signaling, long range $\mathrm{Wg}$ signaling, and presumably also Dpp signaling, plus a preblade fate of the cells (Zecca and Struhl 2007b; Zecca and Struhl 2007a). How does $\mathrm{Vg}$ regulate cell survival and proliferation? It has been described that $\mathrm{Vg}$ interacts with Scalloped (Sd), and the $\mathrm{Vg} / \mathrm{Sd}$ dimer activates the transcription of target genes, such as $v g$ and $s d$ themselves as well as the gene encoding the cell cycle activator dE2F1 (Simmonds et al. 1998; Halder and Carroll 2001). Recently, Sd was found to also dimerize with Yorkie (Yki), a transcription factor acting in the Hippo tumor suppressor pathway, thus leading to the speculation that $\mathrm{Sd}$ acts with $\mathrm{Vg}$ and Yki together to regulate a set of common target genes. Some recent studies (Goulev et al. 2008; Wu et al. 2008) indicate that this might indeed be the case; however, the $v g$ phenotype suggests that it also regulates genes which are not influenced by the Hippo pathway. Certainly more experiments are needed to clarify the link between Vg and Yki. Apart from the evidence for growth regulation via $\mathrm{Vg}$, several experiments show that Dpp regulates proliferation also independently of $\mathrm{Vg}$. Contrary to the overgrowth phenotype of discs lacking brk function or expressing Dpp uniformly, ubiquitous expression of $v g$ does not lead to larger discs (Baena-Lopez and Garcia-Bellido 2006). 
Moreover, although $v g$ is expressed only in the enlarged pouch of brk mutant discs (or discs expressing Dpp uniformly), enhanced cell proliferation also occurs outside of the pouch (Campbell and Tomlinson 1999; Schwank et al. 2008).

Besides the link between Dpp and Hippo via $\mathrm{Vg}$, two further studies suggest that the Hippo pathway might be important for the growth regulatory activity of Dpp. One of them describes an effect of Brk on the expression levels of the Hippo target bantam (Martin et al. 2004), and recent work by Rogulja et al. supports a model in which the Dpp signaling gradient influences the activity of the Fat/Hippo pathway by regulating the expression and localization of Hippo pathway components. This latter study shows that the Dpp gradient controls the expression of the four-jointed $(f j)$ and dachsous ( $d s)$ genes. Graded Fj and Ds levels lead to partial inactivation of Fat and hence decreased Hippo pathway activity, which in turn causes growth (Rogulja et al. 2008). However, $f j$ and $d s$ expression is also influenced by Vg (Baena-Lopez and Garcia-Bellido (2006); Cho and Irvine 2004), and it is possible that Dpp affects the expression of $d s$ and $f j$ only indirectly, via $v g$. Consistent with this view, Baena-Lopez and Garcia-Bellido (2006) showed that graded vg expression is essential to promote normal wing growth. However, not much is known at present about the establishment of graded $v g$ expression along the $\mathrm{P} / \mathrm{D}$ axis. Future studies will have to determine the extent to which graded $v g$ expression is accomplished by morphogen gradients or by other molecular mechanisms, such as the autoregulatory feed-forward loop that causes the expansion of the $v g$ expressiondomain (Zecca and Struhl 2007b; Zecca and Struhl 2007a).

\section{THE DPP GRADIENT AND THE FINAL SIZE QUESTION}

One of the long-standing unsolved issues in developmental biology concerns the mechanism by which the final size of an organ is controlled. Organs show a very characteristic growth pattern, and they stop growing at a definite size. This final size is regulated by organ extrinsic and organ intrinsic factors. Examples for organ extrinsic growth regulators are the insulin-like growth hormones, which can regulate the overall size of an organism. Here, we focus on organ-intrinsic growth control. First evidence for the existence of intrinsic mechanisms came from grafting experiments in salamanders early in the last century. Twitty and Schwind (1931) showed, with species of different size (the small Ambystoma punctatum and the large Ambystoma tigrinum), that grafted limbs adopt the final size of the donor-even when implanted in a differently sized host. Experiments with Drosophila wing imaginal discs also provided evidence for organ intrinsic growth mechanisms. Discs were removed and cultured either in adult or larval hosts, providing them with extra time for growth. Such discs, however, did not overgrow, arguing for an organ intrinsic growth-arrest system (Bryant and Simpson 1984). Knowledge about the molecular nature of such organ intrinsic growth-arrest systems remains fragmentary. In Drosophila, polyploidy or altered expression of cell cycle genes can cause differences in cell size, but these do not alter the final size of wing imaginal discs (Santamaria 1983; Weigmann et al. 1997; Neufeld et al. 1998). These observations argue for a mechanism in which the dimensions of wing discs are measured and not the number of cells they are composed of, because changes in cell number are compensated by changes in cell size and vice versa.

The tight link between patterning and final size control, and the identification of the dual role of morphogens_-regulating not only patterning but also growth-led to the idea that the dimensions of an organ, including those of its end state, might be assessed and governed by morphogen gradients. Dpp has the potential to modulate the final size of the Drosophila wing; $d p p$ hypomorphic mutant animals (carrying $d p p^{\text {disc }}$ alleles) develop only wing stumps; and animals expressing Dpp ectopically within the entire wing disc develop overgrown wing discs (Spencer et al. 1982; Capdevila and 
A Gradient model by Day and Lawrence

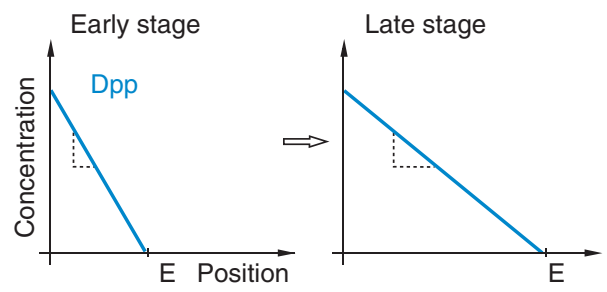

B Mechanical feedback models

B1) by Hufnagel et al.

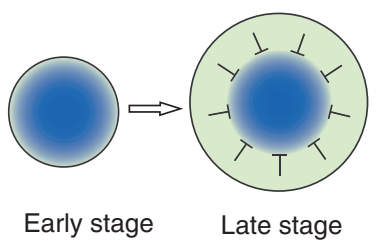

B2) by Aegerter-Wilmsen et al.

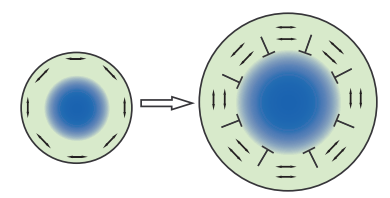

Early stage Late stage

Growth factor $\perp$ Compressing force

- Stretching force

C Inhibitor model by Nijhout

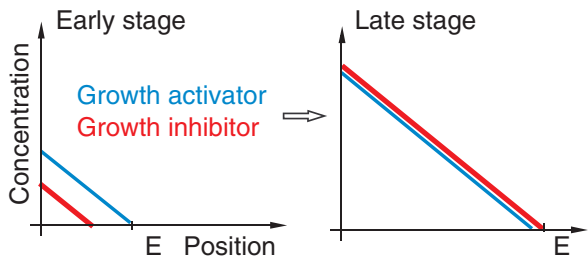

Guerrero 1994; Zecca et al. 1995; Burke and Basler 1996; Martin-Castellanos and Edgar 2002). These properties of Dpp rendered the Drosophila wing an attractive system for researchers to develop and challenge models for the final size control by morphogen gradients. Here, we describe the most prominent of these models, and challenge them with experimental evidence or counter evidence.

The first model discussed here is the gradient model by Day and Lawrence (2000). It
Figure 4. Models for final size control of the wing disc by the Dpp gradient. (A) This model is based on the assumption that Dpp levels in the center and the periphery are fixed, resulting in a decline of the slope of the Dpp gradient when the disc grows. Growth stops when the slope is sufficiently shallow. (B1) This model assumes that the Dpp gradient does not adapt to the disc size. Once the disc has grown, lateral cells do not receive sufficient Dpp and stop proliferating. A mechanical feedback mechanism exerts a compression force to medial cells, leading to a growth stop also in this region. (B2) Growth of the wing disc is governed by a combination of morphogens, which are concentrated at the center of the disc, and stretching and compression forces. High growth factor levels in the center of the disc lead to local growth. This causes stretching, and thus growth in the circumferential regions of the disc, with some stretching still remaining. Stretching forces in the periphery in turn lead to compression of medial cells, which influences growth negatively. As the disc grows, the stretched region becomes wider and compression in the center of the disc becomes stronger. Growth stops once the influence of compression forces becomes bigger than the growth promoting influence by Dpp. $(C)$ In parallel to a growth activator, a growth inhibitor is expressed. Initially, the growth activator is expressed at higher levels. Over time, this ratio changes, and growth stops when the inhibitor reaches a concentration higher than the activator. assumes that the Dpp concentration in the center and at the disc margins are fixed. Thus, when the size of the wing disc increases the slope of the Dpp, concentration gradient decreases. Growth stops when the slope drops below a certain level (Fig. 4A). As already discussed previously, this model would predict that homogenous expression of Dpp would lead to a growth stop, but this is not the case. Moreover, a recent study concluded that the slope of the Dpp gradient does not change 
and stays constant during growth (Hufnagel et al. 2007; Bollenbach et al. 2008).

These findings prompted Hufnagel et al. (2007) to develop an alternative model of size determination. They proposed that once the disc grows to a size in which the morphogen concentration in the boundary region is below a threshold, the boundary cells stop proliferating. Mechanical constraints then lead to compression of cells in the central region. Under the assumption that compression inhibits growth, this would eventually lead to a growth stop within the entire disc (Fig. 4B1). However, experiments in which the $\mathrm{P}$ compartment size was changed via altered Insulin signaling activity showed that the Dpp activity gradient has the ability to compensate for differences in compartment size, and changes the slope (Teleman and Cohen 2000). Clearly, though, for the mechanical feedback model of Hufnagel et al. (2007), it is essential that the Dpp morphogen gradient is constant and independent of disc size.

Another model for final size control based on mechanical forces was developed by Aegerter-Wilmsen et al. (2007). In this model, compression of cells leads to a reduction in proliferation while stretching causes increased proliferation. During wing disc development, Dpp (possibly in conjunction with other morphogens or growth factors) leads to growth in the central region of the disc, which leads to mechanical stretching and thus proliferation in the periphery of the disc. Because the stretching is not completely compensated for by growth, this causes compression and consequently reduced growth in the center of the disc. These compression forces become larger as disc size increases, and once their effects reach an extent that negates the growth promoting activity by Dpp, growth ceases (Fig. 4B2). Measuring mechanical forces in tissues is experimentally very difficult, thus models that rely on mechanical forces have remained speculative so far.

The last model for final size control discussed here was formulated without specific reference to Dpp or imaginal discs (Nijhout 2003), but it can be adjusted to the Dpp gradient in the wing. It suggests that an organ produces its own growth factor (activator), as well as a factor that destroys this growth activator (inhibitor). Initially, the growth activator is produced at a higher amount than the growth inhibitor. During development, this ratio changes, and once the inhibitor reaches a higher concentration than the activator, growth ceases (Fig. 4C). With Dpp acting as an activator in the wing, one would expect the inhibitor to occur in a similar gradient distribution, because the stop of proliferation as well as proliferation during wing disc development happens uniformly throughout the disc. So far, there is no experimental evidence for the existence of such an inhibitor.

In all four models described here, the Dpp gradient is the "key factor" for final size control. However, experiments that have been conducted so far indicate that Dpp is merely one among many growth modulators, and suggest that several other signaling pathways, including the $\mathrm{Wg}, \mathrm{Hh}, \mathrm{N}$, Insulin, and Hippo pathways, also play a role in growth of wing imaginal discs. It is likely that they act together with Dpp to regulate the final size of this organ. One important set of experiments would therefore be to study the effect of all these additional signaling pathways on final size. The termination of growth under normal conditions coincides in time with pupariation and the release of ecdysone, a hormonal signal which affects cell proliferation independently of the organ-intrinsic growth control system. That this systemic, organ extrinsic growth-arrest signal overlaps with the organ intrinsic growth-arrest system is not surprising, and indicates that during development, both processes are tightly connected. However, it complicates the work of biologists who preferentially would first study both processes separately, and then investigate their mutual interference. For example, if one wants to examine how changes in the activity of different signaling pathways influence the organ intrinsic growth control system, the effects might be overruled by the impact of ecdysone on growth-stop. Thus, to study the influence of potential intrinsic regulators of final size, we must 


\section{G. Schwank and K. Basler}

develop protocols for culturing and studying discs outside of the larva, under conditions in which organ extrinsic factors are distributed uniformly over time, and therefore do not trigger a growth arrest. The identification of the molecular components involved in organ size control is likely going to provide the key to being able to mechanistically understand this most fascinating conundrum of biology.

\section{ACKNOWLEDGMENTS}

We would like to thank Peter Gallant, Hugo Stocker, Marko Jovanowic, Simon Restrepo, Maria Willecke, Tinri Aegerter-Wilmsen, and George Hausmann for discussions and comments on the manuscript.

\section{REFERENCES}

Adachi-Yamada T, O'Connor MB. 2002. Morphogenetic apoptosis: A mechanism for correcting discontinuities in morphogen gradients. Dev Biol 251: 74-90.

Adachi-Yamada T, Fujimura-Kamada K, Nishida Y, Matsumoto K. 1999. Distortion of proximodistal information causes JNK-dependent apoptosis in Drosophila wing. Nature 400: 166-169.

Aegerter-Wilmsen T, Aegerter CM, Hafen E, Basler K. 2007. Model for the regulation of size in the wing imaginal disc of Drosophila. Mech Dev 124: 318-326.

Affolter M, Basler K. 2007. The Decapentaplegic morphogen gradient: From pattern formation to growth regulation. Nat Rev Genet 8: 663-674.

Agrawal N, Joshi S, Kango M, Saha D, Mishra A, Sinha P. 1995. Epithelial hyperplasia of imaginal discs induced by mutations in Drosophila tumor suppressor genes: Growth and pattern formation in genetic mosaics. Dev Biol 169: 387-398.

Baena-Lopez LA, Garcia-Bellido A. 2006. Control of growth and positional information by the graded vestigial expression pattern in the wing of Drosophila melanogaster. Proc Natl Acad Sci 103: 13734-13739.

Basler K, Struhl G. 1994. Compartment boundaries and the control of Drosophila limb pattern by hedgehog protein. Nature 368: 208-214.

Beadle GW, Tatum Edward L, Clancy CW. 1938. Food level in relation to rate of development and eye pigmentation in Drosophila melanogaster. Biol Bull 75: 447-462.

Bénazet JD, Zeller R. 2009. Vertebrate limb development: moving from classical morphogen gradients to an integrated 4-dimensional patterning system. Cold Spring Harb Perspect Biol doi: 10.1101/cshperspect.a001339.

Blair SS. 2007. Wing vein patterning in Drosophila and the analysis of intercellular signaling. Annu Rev Cell Dev Biol 23: 293-319.
Bohn H. 1976. Tissue interactions in the regenerating cockroach leg. In Insect development (ed. P.A. Lawrence), Royal Entomological Society of London Symposium No. 8: pp. 170-185. Blackwell, Oxford, UK.

Bollenbach T, Pantazis P, Kicheva A, Bokel C, Gonzalez-Gaitan M, Julicher F. 2008. Precision of the Dpp gradient. Development 135: 1137-1146.

Bryant PJ, Levinson P. 1985. Intrinsic growth control in the imaginal primordia of Drosophila, and the autonomous action of a lethal mutation causing overgrowth. Dev Biol 107: 355-363.

Bryant PJ, Simpson P. 1984. Intrinsic and extrinsic control of growth in developing organs. Q Rev Biol 59: 387-415.

Burke R, Basler K. 1996. Dpp receptors are autonomously required for cell proliferation in the entire developing Drosophila wing. Development 122: 2261-2269.

Campbell G, Tomlinson A. 1999. Transducing the Dpp morphogen gradient in the wing of Drosophila: Regulation of Dpp targets by brinker. Cell 96: 553-562.

Capdevila J, Guerrero I. 1994. Targeted expression of the signaling molecule decapentaplegic induces pattern duplications and growth alterations in Drosophila wings. EMBO J 13: 4459-4468.

Cho E, Irvine KD. 2004. Action of fat, four-jointed, dachsous and dachs in distal-to-proximal wing signaling. Development 131: 4489-4500.

Cook O, Biehs B, BierE. 2004. Brinkerand optomotor-blind act coordinately to initiate development of the L5 wing vein primordium in Drosophila. Development 131: 2113-2124.

Couso JP, Knust E, Martinez Arias A. 1995. Serrate and wingless cooperate to induce vestigial gene expression and wing formation in Drosophila. Curr Biol 5: $1437-1448$.

Day SJ, Lawrence PA. 2000. Measuring dimensions: The regulation of size and shape. Development 127: 2977-2987.

Eldar A, Rosin D, Shilo BZ, Barkai N. 2003. Self-enhanced ligand degradation underlies robustness of morphogen gradients. Dev Cell 5: 635-646.

Entchev EV, Schwabedissen A, Gonzalez-Gaitan M. 2000. Gradient formation of the TGF-beta homolog Dpp. Cell 103: 981-991.

French V, Bryant PJ, Bryant SV. 1976. Pattern regulation in epimorphic fields. Science 193: 969-981.

Gelbart WM. 1989. The decapentaplegic gene: A TGF-beta homologue controlling pattern formation in Drosophila. Development (suppl.) 107: 65-74.

Gibson MC, Perrimon N. 2005. Extrusion and death of DPP/BMP-compromised epithelial cells in the developing Drosophila wing. Science 307: 1785-1789.

Goulev Y, Fauny JD, Gonzalez-Marti B, Flagiello D, Silber J, Zider A. 2008. SCALLOPED interacts with YORKIE, the nuclear effector of the hippo tumor-suppressor pathway in Drosophila. Curr Biol 18: 435-441.

Halder G, Carroll SB. 2001. Binding of the Vestigial co-factor switches the DNA-target selectivity of the Scalloped selector protein. Development 128: 3295-3305.

Hufnagel L, Teleman AA, Rouault H, Cohen SM, Shraiman BI. 2007. On the mechanism of wing size determination in fly development. Proc Natl Acad Sci 104: $3835-3840$. 
Jazwinska A, Kirov N, Wieschaus E, Roth S, Rushlow C. 1999. The Drosophila gene brinker reveals a novel mechanism of Dpp target gene regulation. Cell 96: 563-573.

Kimelman D, Kirschner M. 1987. Synergistic induction of mesoderm by FGF and TGF-beta and the identification of an mRNA coding for FGF in the early Xenopus embryo. Cell 51: 869-877.

Kim J, Sebring A, Esch JJ, Kraus ME, Vorwerk K, Magee J, Carroll SB. 1996. Integration of positional signals and regulation of wing formation and identity by Drosophila vestigial gene. Nature 382: 133-138.

Lawrence PA, Struhl G. 1996. Morphogens, compartments, and pattern: Lessons from Drosophila? Cell 85: 951-961.

Macdonald SJ, Goldstein DB. 1999. A quantitative genetic analysis of male sexual traits distinguishing the sibling species Drosophila simulans and D. sechellia. Genetics 153: $1683-1699$.

Martin FA, Morata G. 2006. Compartments and the control of growth in the Drosophila wing imaginal disc. Development 133: 4421-4426.

Martin FA, Perez-Garijo A, Moreno E, Morata G. 2004. The brinker gradient controls wing growth in Drosophila. Development 131: 4921-4930.

Martin-Castellanos C, Edgar BA. 2002. A characterization of the effects of Dpp signaling on cell growth and proliferation in the Drosophila wing. Development 129: 1003-1013.

Marty T, Muller B, Basler K, Affolter M. 2000. Schnurri mediates Dpp-dependent repression of brinker transcription. Nat Cell Biol 2: 745-749.

Milan M, Campuzano S, Garcia-Bellido A. 1996. Cell cycling and patterned cell proliferation in the wing primordium of Drosophila. Proc Natl Acad Sci 93: 640-645.

Minami M, Kinoshita N, Kamoshida Y, Tanimoto H, Tabata T. 1999. Brinker is a target of Dpp in Drosophila that negatively regulates Dpp-dependent genes. Nature 398: 242-246.

Moreno E, Basler K, Morata G. 2002. Cells compete for decapentaplegic survival factor to prevent apoptosis in Drosophila wing development. Nature 416: 755-759.

Nellen D, Affolter M, Basler K. 1994. Receptor serine/threonine kinases implicated in the control of Drosophila body pattern by decapentaplegic. Cell 78: 225-237.

Nellen D, Burke R, Struhl G, Basler K. 1996. Direct and long-range action of a DPP morphogen gradient. Cell 85: $357-368$.

Neufeld TP, de la Cruz AF, Johnston LA, Edgar BA. 1998. Coordination of growth and cell division in the Drosophila wing. Cell 93: 1183-1193.

Nijhout HF. 2003. The control of body size in insects. Dev Biol 261: 1-9.

Rogulja D, Irvine KD. 2005. Regulation of cell proliferation by a morphogen gradient. Cell 123: 449-461.

Rogulja D, Rauskolb C, Irvine KD. 2008. Morphogen control of wing growth through the Fat signaling pathway. Dev Cell 15: 309-321.

Santamaria P. 1983. Analysis of haploid mosaics in Drosophila. Dev Biol 96: 285-295.

Schwank G, Restrepo S, Basler K. 2008. Growth regulation by Dpp: An essential role for Brinker and a non-essential role for graded signaling levels. Development 135: 4003-4013.

Serrano N, O’Farrell PH. 1997. Limb morphogenesis: Connections between patterning and growth. Curr Biol 7: R186-195.

Shen J, Dahmann C. 2005. Extrusion of cells with inappropriate Dpp signaling from Drosophila wing disc epithelia. Science 307: 1789-1790.

Shraiman BI. 2005. Mechanical feedback as a possible regulator of tissue growth. Proc Natl Acad Sci 102: 3318-3323.

Simmonds AJ, Liu X, Soanes KH, Krause HM, Irvine KD, Bell JB. 1998. Molecular interactions between Vestigial and Scalloped promote wing formation in Drosophila. Genes Dev 12: 3815-3820.

Simpson P. 1976. Analysis of the compartments of the wing of Drosophila melanogaster mosaic for a temperaturesensitive mutation that reduces mitotic rate. Dev Biol 54: 100-115.

Singer MA, Penton A, Twombly V, Hoffmann FM, Gelbart WM. 1997. Signaling through both type I DPP receptors is required for anterior-posterior patterning of the entire Drosophila wing. Development 124: 79-89.

Sivasankaran R, Vigano MA, Muller B, Affolter M, Basler K. 2000. Direct transcriptional control of the Dpp target omb by the DNA binding protein Brinker. $E M B O J$ 19: 6162-6172.

Spencer FA, Hoffmann FM, Gelbart WM. 1982. Decapentaplegic: A gene complex affecting morphogenesis in Drosophila melanogaster. Cell 28: 451-461.

Sturtevant MA, Biehs B, Marin E, Bier E. 1997. The spalt gene links the A/P compartment boundary to a linear adult structure in the Drosophila wing. Development 124: $21-32$.

Teleman AA, Cohen SM. 2000. Dpp gradient formation in the Drosophila wing imaginal disc. Cell 103: 971-980.

Ten Berge D, Brugmann SA, Helms JA, Nusse R. 2008. Wnt and FGF signals interact to coordinate growth with cell fate specification during limb development. Development 135: 3247-3257.

Towers M, Mahood R, Yin Y, Tickle C. 2008. Integration of growth and specification in chick wing digit-patterning. Nature 452: 882-886.

Twitty V, Schwind J. 1931. The growth of eyes and limbs transplanted heteroplastically between two species of salamander. J Exp Zool 59: 61-86.

Van de Bor V, Delanoue R, Cossard R, Silber J. 1999. Truncated products of the vestigial proliferation gene induce apoptosis. Cell Death Differ 6: 557-564.

Weigmann K, Cohen SM, Lehner CF. 1997. Cell cycle progression, growth and patterning in imaginal discs despite inhibition of cell division after inactivation of Drosophila Cdc2 kinase. Development 124: 3555-3563.

Wolpert L. 1969. Positional information and the spatial pattern of cellular differentiation. J Theor Biol 25: 1-47.

Wolpert L. 1981. Cellular basis of skeletal growth during development. Br Med Bull 37: 215-219.

Wu S, Liu Y, Zheng Y, Dong J, Pan D. 2008. The TEAD/TEF family protein Scalloped mediates transcriptional output of the Hippo growth-regulatory pathway. Dev Cell 14: $388-398$. 


\section{G. Schwank and K. Basler}

Zecca M, Struhl G. 2007a. Control of Drosophila wing growth by the vestigial quadrant enhancer. Development 134: 3011-3020.

Zecca M, Struhl G. 2007b. Recruitment of cells into the Drosophila wing primordium by a feed-forward circuit of vestigial autoregulation. Development 134: 3001-3010.
Zecca M, Basler K, Struhl G. 1995. Sequential organizing activities of engrailed, hedgehog and decapentaplegic in the Drosophila wing. Development 121: 2265-2278.

Zhu J, Nakamura E, Nguyen MT, Bao X, Akiyama H, Mackem S. 2008. Uncoupling Sonic hedgehog control of pattern and expansion of the developing limb bud. Dev Cell 14: 624-632. 


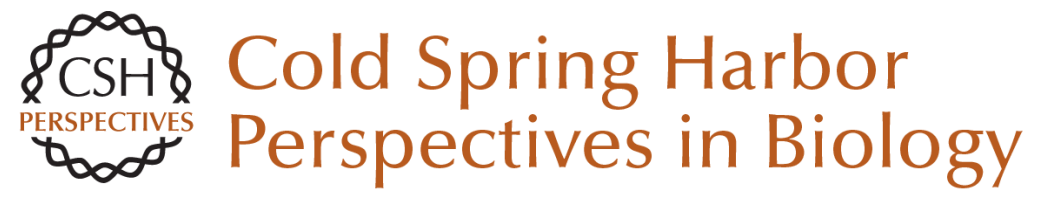

\section{Regulation of Organ Growth by Morphogen Gradients}

Gerald Schwank and Konrad Basler

Cold Spring Harb Perspect Biol 2010; doi: 10.1101/cshperspect.a001669 originally published online July 29, 2009

\section{Subject Collection Generation and Interpretation of Morphogen Gradients}

Regulation of Organ Growth by Morphogen Gradients

Gerald Schwank and Konrad Basler

Signaling Gradients during Paraxial Mesoderm

Development Alexander Aulehla and Olivier Pourquié

Morphogen Gradient Formation

Ortrud Wartlick, Anna Kicheva and Marcos

González-Gaitán

Nodal Morphogens

Alexander F. Schier

Gradients and the Specification of Planar Polarity

in the Insect Cuticle

David Strutt

Vertebrate Limb Development: Moving from

Classical Morphogen Gradients to an Integrated

4-Dimensional Patterning System Jean-Denis Bénazet and Rolf Zeller

Establishing and Interpreting Graded Sonic

Hedgehog Signaling during Vertebrate Neural

Tube Patterning: The Role of Negative Feedback Vanessa Ribes and James Briscoe

Systems Biology of the Self-regulating

Morphogenetic Gradient of the Xenopus Gastrula Jean-Louis Plouhinec and E. M. De Robertis

\section{Gradients in Planarian Regeneration and} Homeostasis

Teresa Adell, Francesc Cebrià and Emili Saló

Shaping Morphogen Gradients by Proteoglycans Dong Yan and Xinhua Lin

Forming Patterns in Development without Morphogen Gradients: Scattered Differentiation and Sorting Out

Robert R. Kay and Christopher R.L. Thompson

Robust Generation and Decoding of Morphogen Gradients

Naama Barkai and Ben-Zion Shilo

Models for the Generation and Interpretation of

Gradients

Hans Meinhardt

Graded Dorsal and Differential Gene Regulation in the Drosophila Embryo

Gregory T. Reeves and Angelike Stathopoulos

Chemical Gradients and Chemotropism in Yeast Robert A. Arkowitz

Gradients in the Brain: The Control of the Development of Form and Function in the Cerebral Cortex

Stephen N. Sansom and Frederick J. Livesey

For additional articles in this collection, see http://cshperspectives.cshlp.org/cgi/collection/

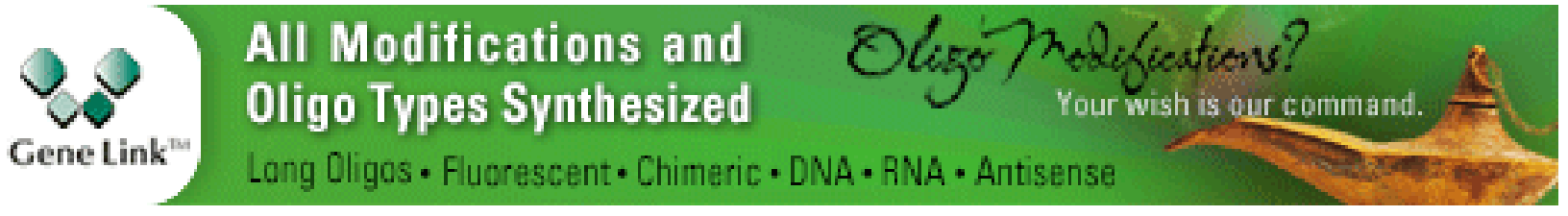


For additional articles in this collection, see http://cshperspectives.cshlp.org/cgi/collection/

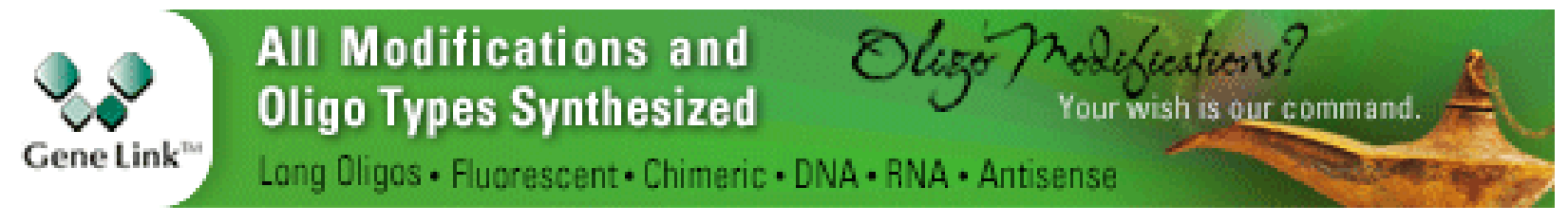

Copyright @ 2010 Cold Spring Harbor Laboratory Press; all rights reserved 